nerve fibres which pass to glands, blood vessels, and nonstriped muscle fibres. The autonomic system (or extended vagus) consists of nerve fibres with peripheral ganglia arranged in plexuses near the viscera to be supplied, the chief of which are the cardiac, solar, and hypogastric plexuses. Stimuli conveyed by the sympathetic and autonomic systems are mutually antagonistic; thus, for example, stimulation of the sympathetic increases pulse frequency, whereas stimulation of the vagus slows the heart; the vagus is motor and secretory to the stomach, and its stimulation causes an increase in the amount and acidity of the gastric juice, contraction of the stomach, and relaxation of the pyloric sphincter, whereas excitation of the sympathetic inhibits peristalsis and contracts the sphincter. It is the balance of these two sets of stimuli which regulates the functioning of the various organs. People may be divided into two groups according to the dominance of their sympathetic or autonomic sensitiveness; the autonomic or vagotonic person is staid and unemotional, with an infrequent pulse, small pupils, deep-set eyes, and cool skin. The sympatheticotonic individual, on the other hand, is emotional and excitable, with frequent pulse, dilated pupils, and warm skin; they are specially sensitive to adrenaline, which intensifies these characteristics. Sympatheticotonia may be relative owing to diminished activity of the vagotonic system, or actual from increased excitability of the sympathetic system, and in like fashion diminution of adrenal function induces vagotonia.

As the clinical syndromes of autonomic and sympathetic stimulation have many symptoms found in definite endocrine disturbance, it is perhaps justifiable to assume a close connexion between them, though it may not be possible to determine whether such symptoms are due to a disturbance which arises primarily in the endocrine glands or in the nervous system. Adrenaline intensifies sympathetic response, and the stiprarenals help to maintain the normal activity of the sympathetic ganglia, whereas the autonomic or vagus system is activated perhaps by the post-pituitary and alimentary glands. It is of interest in this connexion to compare the physiological action of adrenaline and pituitrin. Adrenaline is more rapid in its action, but the effect is of shorter duration; both raise blood pressure; pituitrin strengthens the force without increasing the frequency of the heart beat, adrenaline accelerates the heart. Pituitrin constricts the coronary and pulmonary vessels, on which adrenaline has no effect. Pituitrin dilates the renal vessels and acts as a diuretic, whereas adrenaline constricts the renal artery: Pituitrin stimulates the intestine, bladder, and puerperal uterus; adrenaline inhibits. Pituitrin acts directly on muscle fibre; adrenaline stimulates the sympathetic nerve fibre. Pituitary secretion is increased by the injection of ovarian extract (Dixon).

It may be that many of the so-called functional diseases -that is, diseases in which no structural lesion is demonstrable with the means at our disposal-are due to disturbance of endocrine function; we are accustomed to speak of various " neuroses," which term implies the idea of an underlying instability of the nervous system as the fons et origo mali. But is it the nervous system which is always primarily at fault? We are frequently called upon to treat "gastric neuroses" -cases of dyspepsia in which peptic ulcer, carcinoma, cholelithiasis, or chronic appendicitis can be certainly excluded. The outstanding symptom may be referable to disturbance in the motor, secretory, or sensory function. Vagotonia is associated with a high stomach, vigorous peristalsis; and hypersecretion; sympatheticotonia with a low and often large viscus, delayed emptying, and hyposecretion. Gastric neuroses present varying clinical pictures, but in not a few of the hyperchlorhydric type evidence of vagotonia may be found if carefully looked for, whereas hypochlorhydria is not infrequently associated with thyroid deficiency, and such patients will derive benefit from small doses of thyroid extract given by the mouth.

Neurasthenia is not infrequently a sequel to an infective disease in which the suprarenal glands may have suffered damage with consequent lowering of function, or it may develop after a prolonged period of strain or anxiety which calls in the first place for increased production of adrenaline with subsequent adrenal exhaustion. The irritant effect of air raids or of physical or mental trauma does not find expression in neurosis until endocrine exhaustion occurs-then obsessions, fears, and anxieties gain the upper hand, overflowing the lowered threshold of control; or in another group the adrenal check on the thyroid is lifted and Graves's disease ensues. The neurasthenic is unable to control the functions of the nervous system in a normal manner, and the recognition of disturbed endocrine activity helps in understanding the symptomatology, and restoration to health will coincide with the readjustment of endocrine balance. In many cases there is doubtless a psychic element also, to which great importance has been attached in some quarters, but the elimination of this feature by itself is but rarely sufficient to effect a cure.

\section{Therapeutics of Endocrine Glands.}

The majority of extracts of ductless glands, as at present prepared, have no obvious effect when administered by the mouth. Animal experiment up to the present has showi that tissue extracts in general, when injected intra-. renously, have much the same effect as any other foreign protein, and, with few exceptions, are in no way specific. Glandular extracts which have a definite action when given by the appropriate route are thyroid, parathyroid, pituitary, pancreas, and adrenal; extracts of intestinal glands given by the mouth in cases of atonic constipation may be helpful. But of these, thyroid and pancreatic alone afford true substitution therapy. There is no evidence that any extract other than thyroid and parathyroid is absorbed as such from the alimentary tract; adrenaline, for example, is only employed by the mouth as an astringent or to inhibit peristalsis in the stomach in cases of haematemesis and vomiting, but no "substitution effect" in Addison's disease is obtainable by feeding with or injection of the gland. Yet in adrenaline we have a valuable remedy as a haemostatic in shock, in asthma, and other spasmodic affections such as cardiospasm, urticaria, and angioneurotic oedema. Pituitrin, when injected, in no way replaces hypofunction of the pars nervosa from which it is prepared, but of its value as a stimulant to the failing myocardium, especially in toxic myocarditis, all will bear witness. It is useful also in intestinal distension not obstructive in origin, in asthma, and above all, I am told, in the third stage of labour.

If any advance in therapeutics is to be made with glandular extracts of doubtful action the practice of giving multiple extracts as a sort of blunderbuss, in the hope that one among the many may hit the mark, must be checked. Before organotherapy is employed we must be sure of our target and spend time and thought in charging our gun.

\section{THE DIFFERENTIAL DIAGNOSIS BETWEEN ACUTE THORACIC AND ACUTE ABDOMINAL LESIONS.*}

\author{
BY
}

V. ZACHARY COPE, M.D., M.S., F.R.C.S.,

SENIOR SURGEON TO THE BOLINGBROKE HOSPITAL; SURGEON TO OUT-PATIENTS, ST. MARY'S HOSPITAL, PADDINGTON.

Ir is well known that thoracic disease frequently causes pain which is referred to the abdomen, and most experienced practitioner's will agree that there is no class of case which causes greater anxiety and difficulty in diagnosis than that in which it is doubtful whether the cause of an acute abdominal pain lies in the thorax or the abdomen. It is imperative to make the diagnosis quickly, for a few hours' delay may cost the patient's life, yet a wrong decision to operate may be equally fatal. The bacteriologist and haematologist are of little help at this stage, and the decision has to be made on clinical grounds.

The difficulty in diagnosis lies for the most part in discriminating between conditions immediately above and below the diaphragm-parietal or diaphragmatic pleurisy, pneumonia, cardiac or pericardiac disease on the one hand, and cholecystitis or a leaking duodenal or gastric ulcer on the other. Frequently appendicitis and other diseases arising

* An address delivered before the Willesden Division of the British Medical Association on February 20th, 1924. 
from lower parts of the abdomen mar give rise to doubt as to the possibility of the pain being referred from the lower chest. Since pleurisy and pneumonia are the commonest acute thoracic diseases, most of these remarks will have special reference to them.

A thorough physical examination must be undertaken in every case. Many general practitioners are now doing a great deal of abdominal surgery; will they deem it presumption on a surgeon's part to remind them always to apply the stethoscope to the chest in a case of abdominal pain?

\section{Previous History.}

Since most acute conditions in the upper part of the abdomen arise in or from the alimentary tract, it is common to learn of some warning in the form of either colicky pains, indigestion, constipation, or diarrhoea. In pulmonary conditions there is frequently the history of a previous common cold or chill, or it may be that there has been exposure to infection from others in the same house. It is true that fulminating appendicitis may come without any warning, and a gastric or duodenal ulcer mav perforate without giving a typical history of previous indigestion. Constipation is not of any great significance, since it is common in people who apparently suffer no great discomfort. Diarrhoea is much more frequent with abdominal than with thoracic lesions.

Onset.

The onset of many acute abdominal conditions is accompanied by collapse, which is unusual with pleuropneumonia. More important is the fact that with very few exceptions acute abdominal cases show no ferer at the start, whilst acute pulmonary conditions usually exhibit several degrees of fever from the very beginning. "At the onset of an attack of acute pancreatitis, or immediately after the perforation of a gastric ulcer, or for some hours after the perforation of a gravid Fallopian tube, or during an attack of biliary colic, or at the very beginning of the pain of an acute attack of appendicitis, the temperature is either normal or subnormal; but it is unusual to find the temperature less than three degrees above normal at the onset of pleurisy or pneumonia (except in old people). If at the onset of a case of acute appendicitis the temperature be taken every two hours it will be found to go up slowly till about 1010 F. is reached after about twenty-four hours. Of course, fever is seen in the reaction stage of most abdominal crises. The main exception is acute prelitis. If a patient has acute pain referred to the abdomen and the temperature is $103^{\circ}$ or $104^{\circ}$ at the onset, one naturally thinks of pneumonia and pyelitis, and in most cases one or other lesion will be found. For the temperature to be a guide it is necessary to see the case very soon after the onset of pain. A persistently low temperature with acute abdominal pain is almost always indicative of acute abdominal disease.

The occurrence of a rigor is more common with pleuropneumonia than with abdominal disease, though prelitis again furnishes an exception, and a few cases of appendicitis and biliary infection may be heralded by a rigor.

Vomiting is customary with most acute abdominal lesions, but is less frequent, except perhaps in children, with thoracic conditions. Abdominal pain accompanied by frequent vomiting or retching is nearly always due to an abdominal condition, but many serious abdominal lesions may not cause serious vomiting till late. Facculent vomiting is always due to intestinal obstruction. If romiting occurs at the onset of the illness, then abates, and afterwards recurs, the condition is almost certain to be abdominal. True bilious vomit usually indicates an abcominal lesion.

The character of the pain and the situation of any referred pain are always of importance. In acute abdominal disease the pain is either continuous or occurs in spasms, whilst in pleurisy the pain is more frequently felt on taking a breath. One must, however, also remember that the pain of a leaking duodenal ulcer is worse on taking a breath, and that biliary colic sometimes causes inhibition of the diaphragmatic movement. If pain which starts in the upper abdomen shifts later to the right iliac fossa or hypogastrium one can count on an abdominal origin. In chest conditions there is frequently tenderness on pressure over the chest wall over the lesion. Cough is usually indicative of thoracic disease.
Examination of the Patient.

It is frequently possible to tell the origin of the trouble as one approaches the bedside. Flushed cheeks witi herpes on the lips and an obvious working of the alae nasi sufficiently indicate a pulmonary condition, whilst a pale or livid face with an anxious expression and sunken eres usually mean a late stage of an abdominal catastrophe. But a doubtful case gives no such obvious indications. The alae nasi should always be carefully watched, for a slight respiratory movement there is frequently seen in the very early stage of pleurisy or pneumonia even when few or no signs are present in the chest. It is very unusual for the alae to be seen moving in the early stage of acute abdominal lesions, though such an action may occur with a lesion, such as an acute subphrenic abscess, which impedes the diaphragm and causes basal lung congestion.

The position which the patient assumes in bed may assist in diagnosis. If the dorsal decubitus be assumed and one or both legs be drawn up there is generally an abdominal lesion. With pleurisy or pneumonia the lateral decubitus is more common, whilst with acute cardiac conditions the patient may prefer to be propped up in bed. If the skin feels dry and hot to the touch, indicating considerable elevation of temperature, the chest and urine should be particularly examined. With severe abdominal colic, or at the onset of fulminating abdominal lesions, the skin mar be cold and clammy, though this passes off as the reaction occurs. The value of the temperature may be summed up as follows: A normal cr subnormal temperature is generally indicative of an abdominal lesion; a high temperature usually points to the chest or kidneys as the source of disease; a moderato degree of fever is of no help in differentiation.

Fxamination of the pulse is not alwars of great ralue. If it be normal in frequency, volume, and regularity, any accompanying abdominal pain is usually abdominal in origin. The reaction stage of many acute abdominal conditions is accompanied by a normal pulse. When the pulse rate is rapid more information is to be gained from its volume, for a small rapid pulse is more frequently met with in peritonitis. The respiration rate is generally increased to a greater extent in thoracic lesions, but it is of importance to remember that rigidity of the abdominal wall is frequently accompanied by a compensatory increase in the rate of respiration. Of greater value is the ratio between pulse and respiration. Normally about 4 to 1 , this ratio is reduced considerably in pneumonia, and a ratio of 2 to 1 almost always indicates pulmonary disease. In the later stages of peritonitis the impeding of the diaphragm may cause a great increase in rate of respiration, but it is common to find a pulmonary lesion-basal congestion or pulmonary collapse-in such cases.

In abdominal disease it is usual to find that the parietal muscular wall is held rigid over the inflamed part, whilst in pulmonary conditions the chest wall on the affected side does not move so well on respiration. Abdominal rigidity cannot always be interpreted so simply. It is well known that the muscles of the abdominal wall may be very rigid as the result of pleuropneumonia, whilst it is very common to have serious intra-abdominal disease with a flaccid abdominal wall. Rigidity may be tested in two ways-either by feeling the resistance with the applied hand or by watching the respiratory movement. The first method is most commonly practised, and, generally speaking, definite and persisting rigidity of any segment of the abdominal wall to the applied hand indicates underlying disease. The more limited the rigidity the more localized the disease. If the rigidity be due to thoracic disease it may be found possible to overcome it by patient palpation, but in the case of abdominal disease conitinued palpation over the inflamed area is likely to increase tio stiffness, since the tenderness is greater the deeper the paipation.

Inmobility of the abdominal wail on respiration is of great value in diagnosis. This chiefly applies to the lower abdomen, for the upper abdominal wall is usually immobile when there is an inflamed area either immediately above or close beneath the diaphragm. When only the lower abdomina! wall is rigid there is usually hypogastric peritonitio; in such a case a characteristic condition is often secil in which on inspiration the region below the umbilicus 
appears to recede. Normally, and especially in the male, the lower abdomen is protruded during inspiration along with the upper abdomen. When the hypogastric wall is rigid the upper abdomen is still pushed forward when a breath is taken, but the stiffening of the lower abdominal wall on the increased intra-abdominal pressure causes an apparent recession. Rigidity of the diaphragm, as indicated by immobility of the upper abdomen, is seen with both supra- ard sub-phrenic conditions, so that diagnosis between the two has to depend on other symptoms.

I attach greater value to phrenic shoulder pain or pain referred to the top of the shoulder owing to irritation of the terminations of the phrenic nerve in the diaphragm. The phrenic comes from the third, fourth, and fifth cervical nerves, but chiefly from the fourth. Irritation of the terminations of the phrenic nerve may cause referred pain over the area of the shoulder supplied by the descending cutaneous branches of the third and fourth nerves. This pain is usually referred to the shoulder on the same side as the lesion. The pain is generally felt in the supraspinous fossa if the back of the diaphragm be irritated, in the clavicular region if the front part of the muscle be affected. The cleltoid and acromial region may be affected by any lesion of the dome. The importance of this statement lies in the fact that certain parts of the diaphragm are more likely to be affected by particular lesions. When a duodenal ulcer perforates, the region of the right crus is irritated, and pain is felt in the right supraspinous fossa. Perforation of an ulcer on the anterior surface of the stomach is likely to irritate both domes of the muscle and thereby cause bilateral shoulder pain. Such a bilateral pain is seldom found with thoracic disease. On the other hand, diaphragmatic pleurisy often causes pain in the clavicular and subclavicular region. This is, I think, due to the fact that friction is more likely to persist in the anterior part of the cul-de-sac between chest wall and diaphragm, for fluid would tend to gravitate posteriorly and prevent friction. Whether this explanation be true or not $I$ have been observing carefully for the past three years, and have so far found that nearly every case of abdominal pain with accompanying subclavicular pain had its origin above the diaphragm. This I consider an important diagnostic point not previously noted. As an example of the diagnostic value of this point take the following:

Mrs. C., aged 50, who for a week had been troubled with a cold in the head and pains in the limbs, was seized with sudden pain in the right hypochondrium on December 30th, 1922. At the same lime she experienced pain on top of and in front of the right shoulder, including the subclavicular region. Vomiting occurred on several occasions. The temperature on December 31 st was $100^{\circ}$ the pulse 120 , but there was no dullness on percussion of the chest, nor could any adventitious sounds be heard. There was a slight cough. Tenderness in the right hypochondrium was elicited, but there was no rigidity. Nothing abnormal was detected by the rectum. The alae nasi were noted to be moving. Chiefly on the situation of the shoulder pain diaphragmatic peurisy $u$ as diagnosed. "I can hear a short catchy rub at the right base." Recovery was uninterrupted.

Cutaneous hyperaesthesia due to visceral disease has often proved of value in diagnosis, but its reliability is by no means absolute. Areas of hyperaesthesia above the umbilicus may be caused by reference from the lower thorax or upper abdomen, but so far as I have observed (in many hundreds of cases) hrperaesthesia of the skin below the level of the umbilicus is always of abdominal origin. The intestines and peritoneum are innervated from the tenth, eleventh, and twelfth dorsal nerves, and disease of those viscera may cause increased sensitiveness of the skin in any part of either iliac triangle. I consider this a very valuable differentiating point. It was of use in the following case :

A man was admitted to St. Mary's Hospital in February this year compaining of pain on the right side of the abdomen. He said that a few days previously he had coughed up some blood. Examination showed the scar of a large gunshot wound at the back of the right lower chest. There was tenderness but no definite rigidity in the right iliac fossa and hyperaesthesia to pin-strite extending upwards from Poupart's ligament for two or three inches. There were râles in the chest, but no definite sign of pneumonia. There were râles in the chest, but no definite sign of pneumonia. to be abdominal (and probably appendicular) on account of the position of the hyperaesthesia and also because there was slight pain in the testicle. An anaesthetic was administered, but this provoked such a severe haemoptysis that it was stopped and the patient sent back to bed.' In three days' time the right spermatic

cord swelled and a lump could be felt in the iliac fossa. This was incised under local anaesthesia and a large abscess evacuated and drained. The abdominal condition did well but the chest symptoms became worse. $X$-ray examination showed much shrapnel in the chest. Bronchopneumonia caused death in two weeks. Autopsy showed that the abdeminal lesion had been a retroperitoneal showed that the abdcminal lesion had
abscess, for which no cause was discovered.

There are several other little devices by which it may be proved that abdominal pain is due to abdominal disease. With a unilateral pain of thoracic origin there is usually no tenderness on the other side of the abdomen and no pain is elicited by pressing the fingers deep in the unaffected side; with abdominal disease such pressure causes pain, since it affects the diseased focus.

There are two muscular tests which, if positive, prove the presence of intra-abdominal disease-the psoas and the obturator tests. They depend upon the ract that when a muscle is moved pain is caused if there be any contiguous inflammatory condition. If pain be felt in the iliac fossa upon flexion or extension of the corresponding thigh, it may be assumed that there is an inflammatory condition adjacent to the psoas muscle. Sometimes hyperextension of the thigh is needed to bring about the pain. The obturator test is performed by rotating the flexed thigh internally; this puts the obturator internus on the stretch, and pain results if there be a contiguous pelvic abscess. Neither of these tests is positive in abdominal pain due to thoracic disease. Another symptom which is never produced by thoracic disease is pain referred to the testicle.

Finally, a rectal examination is often of the greatest value in determining abdominal disease, for tenderness of the pelvic peritoneum or a bulging in that region cannot be simulated by thoracic disease.

The guides I have given are meant for those doubtful cases where the signs in the chest are equivocal or in which it is possible that disease is present in both cavities. In this connexion we must emphasize the unfortunate fact that not infrequently an abdominal disease leads quickly to pulmonary complications, and there are certain cases in which disease starts simultaneously in both thorax ard abdomen.

A general rule I have formulated for myself as the result of some bitter experiences is that if, after thorough examination of both chest and abdomen, doubt remains as to the site of the disease it is generally in the abdomen.

I append a comparative table of symptoms :

AвDominal.

Previous History : Onset : constipation; diarrhoea.

Acute without fever (except pyelitis).

Rigor unusual (except pyelitis).

Vomiting usual.

Pain often shifts downward.

Common cold or " chill "; exposure to infection.

Acute with fever at start.

Rigor common.

Vomiting less common.

Pain thoracic as well as abdominal.

Examination.

Appearance :

Varies from normal to Cheeks flushed; alae nasi work. " abdominal " facies.

ing; sometimes herpes on lips.

kin:

Cold or clammy or normal.

Pulse-Respiration

No sure guide.

Abdominal Wall:

May be rigid.

Phrenic Shoulder Pain

Common, but seldom below clavicle.

Skin Hyperaesthesia :

Common.

Psoas Tost

Often positive.

Obturator Test :

Sometimes (rarely) positive.

Testicular Pain:

Sometimes present.

Rectal Examination:

May elicit tenderness or demonstrate lump.

Examination of Chest :

Frcquently slight râles in May be a rub or dullness or upper abdominal lesions.
Skin may be hot and dry.

Pulse-respiration ratio lessened.

Less commonly rigid.

Common, especially below clavicle.

Rare, and never below level of navel.

Always negative.

Always negative.

Never present.

Negative. sometimes nothing definite at onset of symptoms. 